\title{
Hacia la library 2.0: blogs, rss y wikis
}

\author{
Por Leticia Paula Dobrecky
}

\begin{abstract}
Resumen: Uno de los ejes principales de la denominada web 2.0 es el rol participativo que adquiere el usuario, utilizando nuevas tecnologías que le permiten consumir, generar y gestionar los contenidos en un ambiente de colaboración e interacción social. Repensar los servicios de la biblioteca en base a estos principios fundamentales implica el surgimiento de la library 2.0. Se describen en forma general algunas herramientas que generan una intervención directa de los usuarios, como los blogs, la sindicación de contenidos y las wikis. Además, se comentan sus posibles aplicaciones en las bibliotecas y se analizan algunos casos concretos.
\end{abstract}

Palabras clave: Web 2.0, Library 2.0, Bitácoras, Blogs, Wikis, Sindicación de contenidos, RSS.

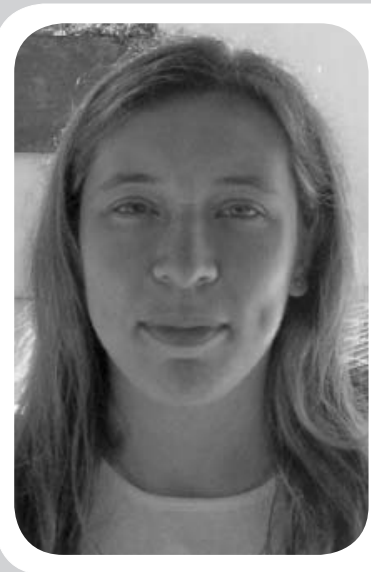

Leticia Paula Dobrecky, es licenciada en bibliotecología y ciencia de la información con orientación en servicios y recursos de información, Facultad de Filosofía y Letras de la Universidad de Buenos Aires. Desde 2002 hasta 2003 participó en un proyecto de actualización de una web en la Cátedra de Fuentes de Información en Humanidades y Ciencias Sociales de dicha Facultad. Durante 2003 trabajó en el Centro de Documentación e Información del Ministerio de Economía y Producción. Actualmente trabaja en el Centro de Documentación e Información Agropecuaria de la Secretaría de Agricultura, Ganadería, Pesca y Alimentos de la Nación (Argentina)

\section{Title: Towards Library 2.0: blogs, rss and wikis}

Abstract: The user participation is one of the principal keys in the web 2.0. The new technologies allow people not only to consume but also create and manage the content in a collaborative environment. The implementation of these new trends in the library context leads to library 2.0. Blogs, rss and wikis, are described. Also, possible applications in libraries are commented and some cases are analyzed.

Keywords: Web 2.0, Library 2.0, Blogs, Wikis, Rss.

Dobrecky, Leticia P. “Hacia la library 2.0: blogs, rss y wikis”. El profesional de la información, 2007, marzo-abril, v. 16, n. 2, pp. 138-142.

DOI: 10.3145/epi.2007.mar.09

\section{Descripción y aplicaciones}

EXISTE UNA VARIEDAD de herramientas que se utilizan como medios de interacción y que propician la participación directa de los usuarios dentro de la conocida como web 2.0 y su vertiente bibliotecaria, la llamada biblioteca 2.0.

\section{Blogs}

Su principal característica es su actualización frecuente y que contiene entradas breves (opiniones, sugerencias, enlaces), en orden cronológico inverso. Existen varios tipos: Ricardo Herrera presenta una clasificación y los divide según a) autoría, b) contenido y c) formato:

a) Individuales (una persona publica los contenidos reflejando sus impresiones y personalidad), los colectivos (hay un administrador que se encarga de coordinar la información y cuenta con colaboradores que participan enviando sus anotaciones y/o comentarios) y los corporativos (las instituciones lo utilizan como boletín de noticias y debates sobre procedimientos).

b) Temáticos (especializados en algo concreto), los misceláneos y los metablogs (versan sobre otros o sobre la blogosfera en general).

c) Fotoblogs (dedicados al mundo de la fotografía), los vblogs (vídeos), los audioblogs (con ficheros audio) y los moblogs (utilizan dispositivos móviles).

Los blogs presentan algunos elementos en común. El cuerpo principal lo constituyen las entradas o anotaciones que se ubican en la parte central y que cuentan con diferentes datos como: fecha, título, texto pro- piamente dicho, categoría, autor, número de comentarios, fecha y hora, enlace fijo y retroenlaces (trackbac$k s)^{1}$. Las entradas se van archivando por orden cronológico, y se muestra un listado con los posts recientes. Normalmente tienen un blogroll que es una lista de enlaces externos a otros recursos relacionados o que el autor considera de interés. Con frecuencia incluyen la posibilidad del rss y un buscador interno.

Tienen múltiples ventajas, siendo las más importantes (junto con el almacenamiento de las entradas en el archivo y la búsqueda por temas) su facilidad de elaboración y mantenimiento, ya que no son necesarios conocimientos técnicos: se basan en un diseño automatizado con plantillas. Las personas que tienen intereses comunes se ponen en contacto a través del blog y entablan "conversaciones". 
Una aplicación de los blogs es la publicación de las novedades de la biblioteca y la promoción de sus servicios y/o productos. Se está comenzando a vislumbrar su importancia en el ámbito educativo para llevar a cabo proyectos de investigación. En este sentido las bibliotecas pueden proporcionar pautas para que los usuarios puedan aprender a utilizar correctamente los buscadores, citar fuentes, localizar y seleccionar recursos de información y sugerir temas. En el servicio virtual de referencia se podrían aprovechar para responder las consultas de los usuarios de un modo colaborativo y conformar una gran base con las preguntas temáticas que se efectúan con mayor frecuencia.

En el plan de desarrollo de la colección, los bibliotecarios deben analizar si los blogs, a veces utilizados para responder las consultas, podrían formar parte del catálogo o bien incluirse en la lista de recursos de internet. No menos importante es su aprovechamiento en la comunicación interna y en la gestión del conocimiento. Por un lado, se podrían publicar informes, boletines, trabajos y establecer conexiones entre los diferentes sectores dentro de la organización. Por el otro, es ideal para crear un repositorio institucional almacenando y organizando el conocimiento tácito y explícito, ordenado tanto en forma cronológica como temática.

En Argentina, la biblioteca de la Universidad Nacional de San Martín (figura 1) elaboró varios blogs en el marco de la denominada Biblioteca Pública Digital, cuyo objetivo es crear un espacio en línea de libre acceso para consultar recursos seleccionados de internet como bases de datos, enciclopedias, bibliotecas digitales y material bibliográfico en texto completo. Se pueden conocer las novedades sobre educación, proyectos de investigación, congresos, eventos, trabajos y servicios a través de Novedades BDigital. Cabe agregar que, dentro del menú de capacitación, se puede acceder al blog Cursos con datos sobre los mismos (presenciales, en línea y módulo autoasistido). A través de los enlaces se puede localizar el módulo de capacitación en línea que funciona como blog y sirve de guía introductoria a diferentes temas, como acceso a bases de datos. Las blogs presentan elementos comunes: entradas organizadas por categorías, enlaces relacionados, motor de búsqueda, archivo, suscripción por rss, posibilidad de enviar comentarios y una guía para crear un blog propio y un canal rss.

Uthink: blogs at university libraries (figura 2) elaborado por la biblioteca de la Universidad de Minnesota tiene como objetivo crear un ambiente para el intercambio de ideas y opiniones y en el que las personas pueden conectarse a lo largo del país. Los alumnos, profesores y el personal de la universidad, pueden crear sus propios blogs que van a estar alojados en el servidor de la institución utilizando el programa Movible Type.

Uno de sus aspectos sobresalientes y destacables es que presenta información muy completa sobre las diferentes posibilidades de implementación de esta herramienta en función de los diferentes tipos de usuarios y se mencionan ejemplos concretos. Por otro lado, es importante destacar su función "educativa" porque orienta y da ayuda y/o guías para que cada persona pueda iniciar su propio blog y promocionarlo. Otra característica no menos importante es que es posible acceder a una wiki dedicada al tema, resultando de utilidad para obtener más respuestas en un entorno de colaboración. Allí, cualquier usuario registrado puede agregar y/o modificar el contenido. Por último, para conocer en profundidad se puede visitar el "reglamento" que describe otros elementos del servicio (derecho de autor, costos, acceso a la tecnología, entre otros).

\section{Sindicación de contenidos}

El rss (rich site summary y sus acrónimos en inglés: really simple syndication, rdf site summary) es parte de la familia de los formatos xml. El objetivo es la distribución masiva de información (noticias) contenida en diferentes sitios. $\mathrm{La}$ mayoría de los autores definen un archivo rss como una descripción

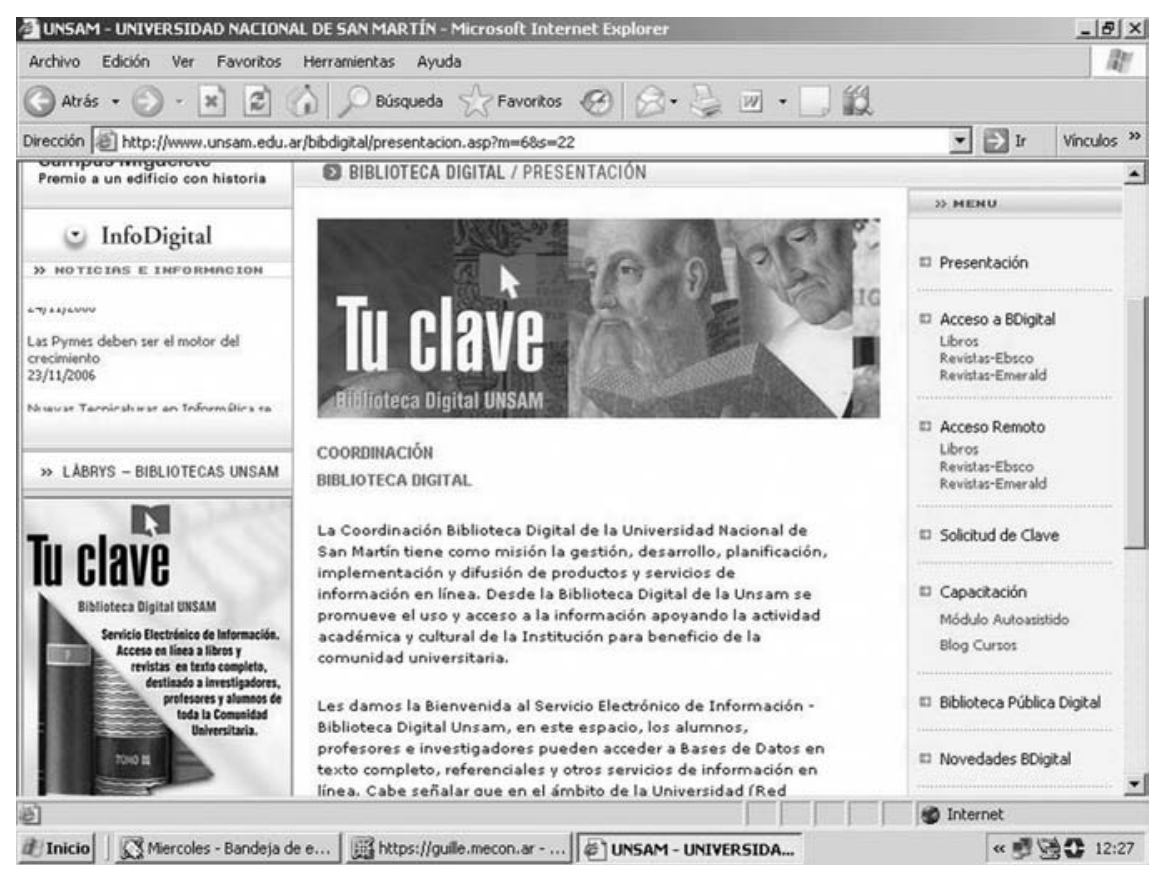

Figura 1.

http://www.unsam.edu.ar/bibdigital/servicios.sp? $m=6 \& s=22 \& s 1=93$ 


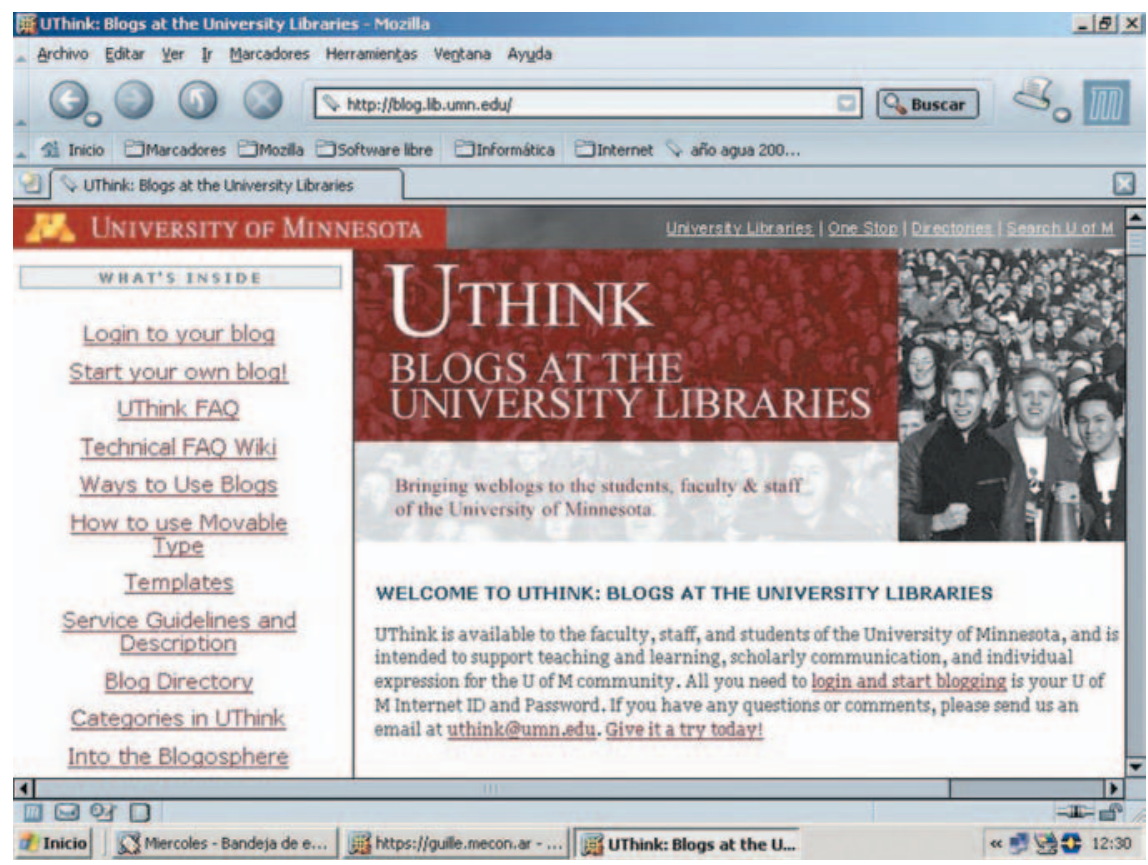

Figura 2.

http://blog.lib.umn.edu/

estructurada (especie de resumen) de uno o varios sitios web. Para poder interpretar estos archivos es necesario contar con programas agregadores. Existen diversas opciones para la lectura de los mismos, algunas están incorporadas en los navegadores, otras están disponibles en línea y otras se tienen que instalar.

Rodrigo Donoso explica la diferencia entre sindicar y agregar. El primero es el acto de poner a disposición de otros el contenido; y el segundo significa absorber de fuentes de contenidos los archivos rss para visualizarlos en nuestro agregador.

Esta técnica adquirió mayor difusión al proliferar los blogs y permite recibir (y ofrecer) información actualizada a modo de una suscripción SDI (difusión selectiva de la información).

Zeki Çelikbaş describe algunas ventajas de la sindicación:

\section{"El rol participativo del usuario es uno de los ejes fundamentales de la web 2.0"}

- privacidad, porque para suscribirse no es necesario enviar un correo electrónico;

- protección contra la publicidad no solicitada, y

- facilidad para cancelar la suscripción.

Una forma interesante de SDI es el que proporciona la National Library of Medicine a través de
Entrez-Pubmed. Los usuarios que utilizan este servicio tienen la posibilidad de recibir las novedades y actualizaciones en su propia computadora en base a su perfil (i. e., estrategia de búsqueda). Se deben seguir algunos pasos que se detallan a medida que se avanza con el procedimiento. Es fundamental contar con un programa agregador para poder recibir la información. Lo más importante es que una vez efectuada la búsqueda es necesario seleccionar del menú "Send to RSS feed" (figura 3). De este modo se accede a una pantalla con opciones para nombrar al canal y limitar el número de registros que deben desplegarse. Luego se crea el feed y se continúa según las instrucciones del sistema.

\section{Wikis}

Aplicación web donde los usuarios registrados de una comunidad pueden crear nuevas páginas o editar las existentes. Es otra forma de colaboración en línea que favorece el intercambio de ideas y el trabajo conjunto en forma continua. Meredith Farkas describe sus componentes principales: el área de discusión para cada página, el historial

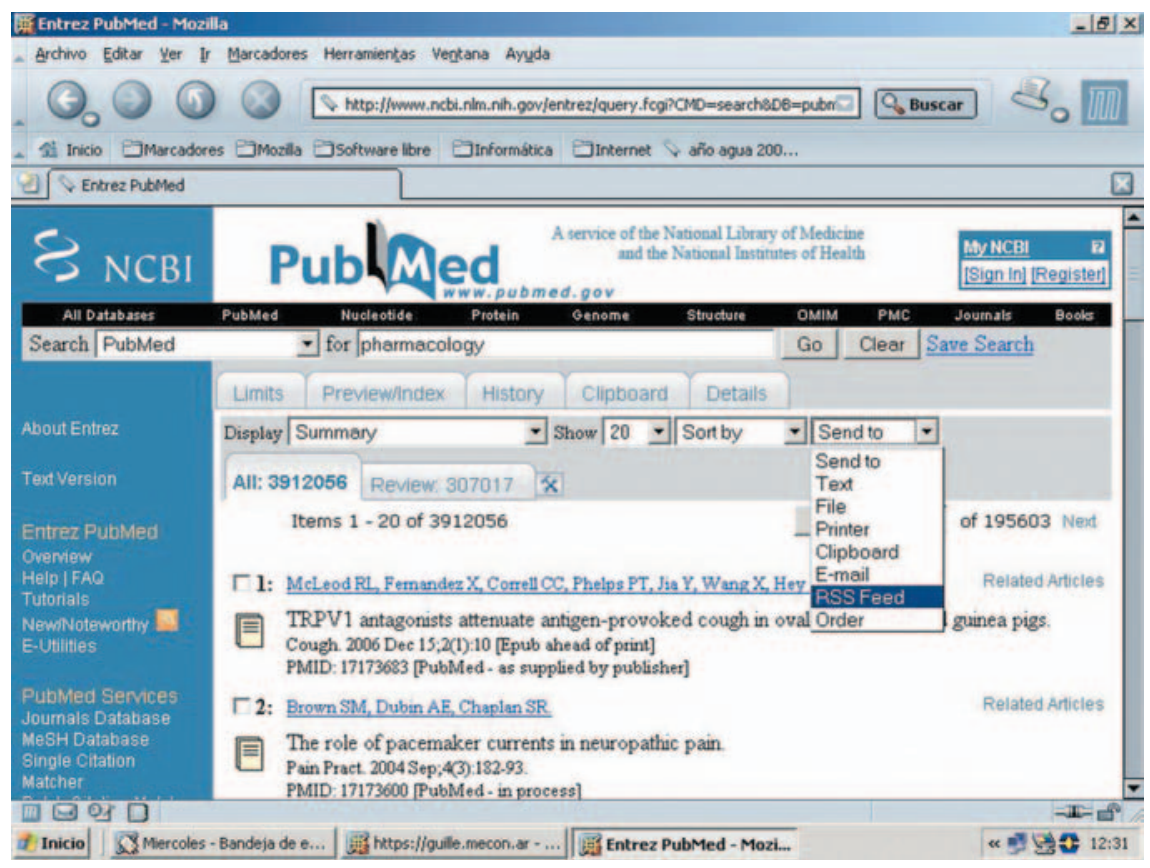

Figura 3.

http://www.pubmed.gov 
que almacena todos los cambios realizados a una de ellas y el listado con todas las modificaciones efectuadas a todas las páginas. Además, se pueden editar y agregar otras y en ocasiones incluyen un buscador.

$\mathrm{Su}$ principal ventaja es la flexibilidad y la facilidad de elaboración. Es ideal para que pequeños grupos de investigación intercambien ideas, produciéndose un ambiente creativo fuente de nuevos desarrollos.

Tiene el riesgo de la falta de control sobre el contenido ya que cualquier persona puede editar los artículos con información errónea. Sus defensores argumentan que el contenido se depura de forma colaborativa ya que si alguien detecta un error tiene la posibilidad de corregirlo. Algunos sistemas tienen distintos niveles para el acceso a la lectura y edición.

\section{"Las nuevas formas de interacción favorecen el intercambio de ideas y contenidos en un marco de colaboración"}

Farkas compara los blogs y las wikis. En los blogs el autor es el que incorpora las entradas que son permanentes, sólo él realiza modificaciones y las personas participan enviando sus comentarios. En las wikis nadie es el "propietario" del contenido, las personas pueden editar los artículos y es un trabajo en colaboración y progreso continuo.

Una de las aplicaciones más frecuentes es para la elaboración de las guías por materias (subject guides) de forma más interactiva y más sencilla de actualizar ya que las personas participan directamente agregando recursos y corrigiendo otros. Una forma novedosa de incorporar funcionalidad al catálogo en línea es que los usuarios participen en el mismo ya sea añadiendo y/o editando el registro y también incorporando reseñas de libros. Además se puede utilizar como un repositorio que almacene el conocimiento colectivo sobre un tema. En el marco de la comunicación interna, para la elaboración de una intranet, para la difusión de las políticas, los manuales a toda la organización; en el desarrollo de proyectos grupales ya que es por excelencia un espacio para el trabajo en colaboración; en el contexto de un evento (por ejemplo una conferencia) para obtener otro estilo de información sobre la ciudad, sobre la conferencia, entre otros.

La Universidad de Ohio desarrolló Biz Wiki (figura 4) dedicada a brindar recursos de información como libros de referencias, bases de datos, sitios web y guías de investigación sobre negocios. Chad Boeninger es el responsable de su elaboración. La idea es que los usuarios puedan localizar la información de un modo más amigable que las tradicionales guías temáticas.

Incluye dos clases de artículos: los recursos y las guías de investigación para temas específicos. Para

\section{"Las nuevas herramientas se caracterizan por su simplicidad y por ser intuitivas"}

buscar se navega por las diferentes categorías y sus correspondientes subcategorías. Un elemento interesante es "Research how-to's" cuya función es orientar a los investigadores a través de guías que muestran paso a paso cómo localizar la información específica de la temática. Mediante el botón "Go" busca en los títulos de los artículos y con "Search" consulta la totalidad de la wiki.

Todas las personas que lo deseen pueden añadir y/o editar el contenido libremente, previa creación de una cuenta.

Jane Dysart y Meredith Farkas desarrollaron para Information Today el "IL 2006 planning wiki" (figura 5) con motivo de la celebración de Internet Librarian en Monterrey, California, para que las personas compartan información sobre dicho evento y la ciudad, preguntar, mantenerse actualizado y establecer

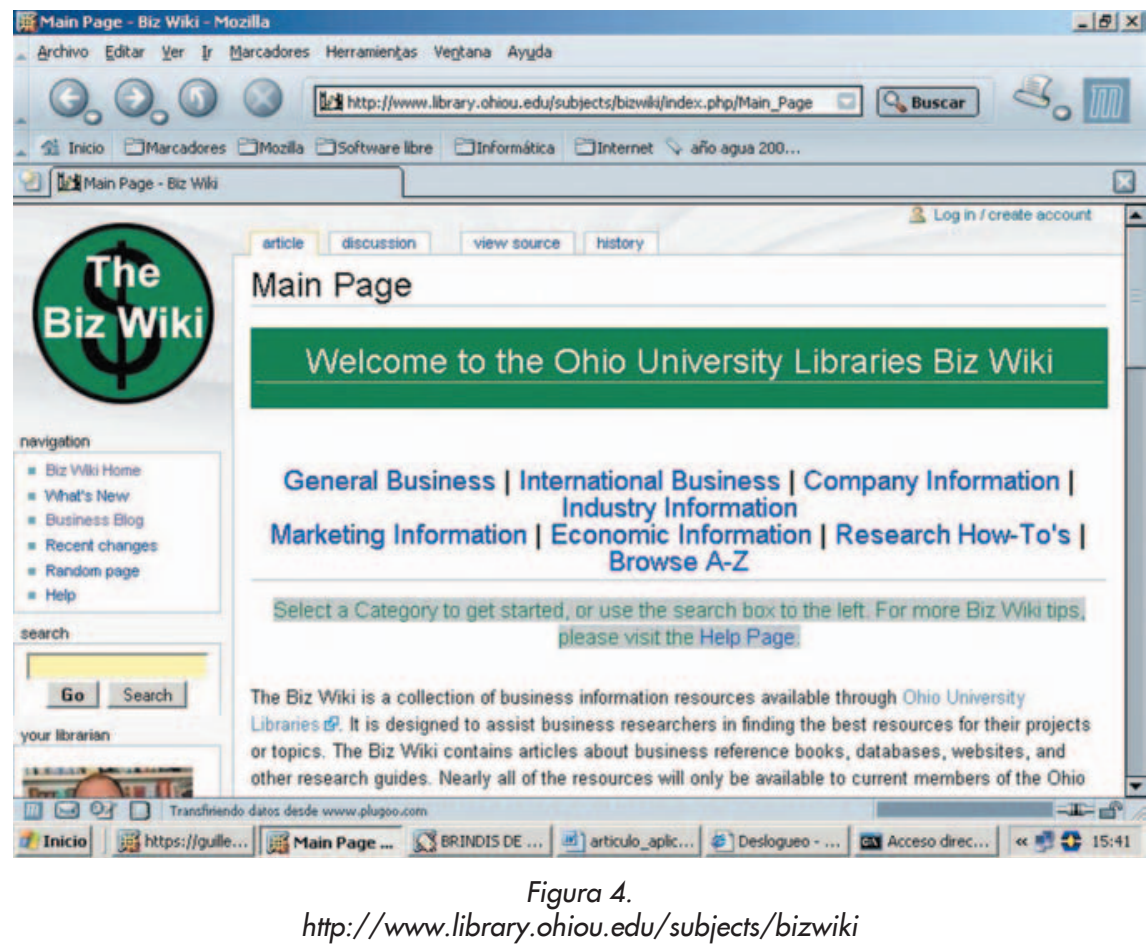

El profesional de la información, v. 16, n. 2, marzo-abril 2007 


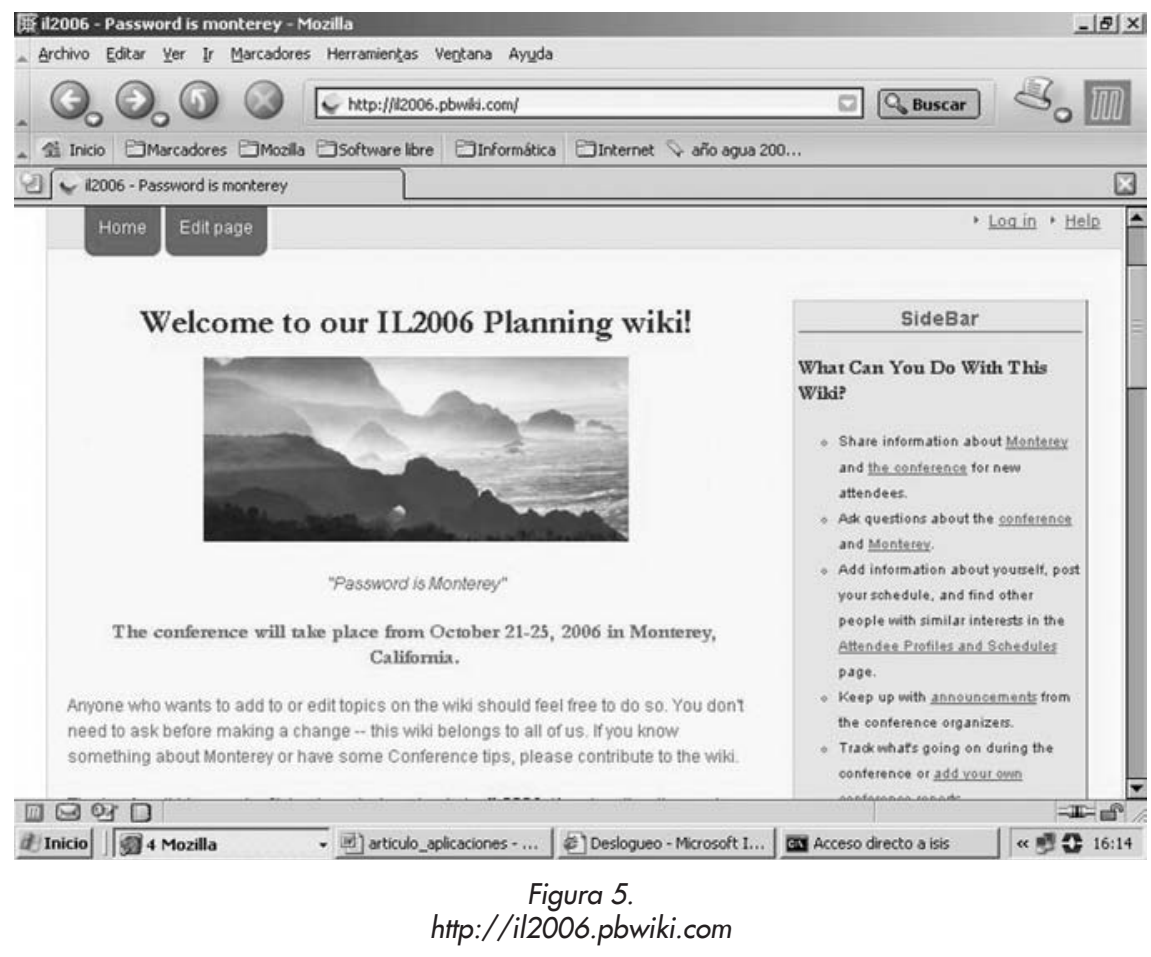

contactos. Está organizada en categorías: noticias, datos sobre la conferencia (anuncios, página oficial de $I L$, sesiones regulares, otros), la ciudad (hoteles, restaurantes, zonas wifi, otros) y para el seguimiento de la misma (informes, recuperar posts sobre el evento por medio de Technorati, etc.).

Cualquiera puede editar el contenido, crear páginas nuevas, etc.

\section{"La library 2.0 implica repensar los servicios de las bibliotecas en función de un usuario más activo"}

\section{Conclusiones}

Las herramientas descritas permiten una interacción directa con los usuarios. El número de blogs aumenta vertiginosamente porque son recursos económicos y simples de elaborar, pero es fundamental contar con los recursos humanos necesarios para actualizar el contenido periódicamente. La sindicación de contenidos es un excelente canal de distribución de noticias. Las wikis no han tenido tanta difusión debido a los peligros que encierra la libertad en la inclusión de contenidos que puede ir en detrimento de su calidad.

Cada institución tendrá que analizar su situación en función de sus posibilidades económicas, tecnológicas y humanas antes de decidir si implementar algunas de estas nuevas tecnologías. Hay que considerar que también pueden aplicarse en el marco de la comunicación interna.

Existen opiniones antagónicas en torno al término library 2.0. Básicamente, se puede decir que los críticos de esta nueva "etiqueta" producto del marketing sostienen que la ideas propuestas no son novedosas, salvo por la incorporación de nuevo software. En cambio los defensores afirman que se ha revolucionado el mundo bibliotecario. Más allá de los debates que se generen, lo importante es conseguir que los usuarios sean partícipes activos en los servicios y productos.

\section{Nota de la Redacción:}

1. Un trackback o enlace inverso es como una cocitación. Cuando se recibe un enlace a algún post del blog, si tiene la posibilidad de poner trackbacks significa que se pueden poner los enlaces que se hayan detectado a través de las estadísticas o los medios automáticos que ofrezca el blog, o que se encuentren por cualquier otro medio (Google, Technorati, etc). Así se produce una retroalimentación. El post del blog A enlaza al post del blog B, y en el post del blog B el trackback enlaza al post del blog A. Así ambos blogs quedan mutuamente enlazados y los visitantes que lleguen a ambos blogs pueden beneficiarse de los enlaces y descubrir nuevas fuentes (Francisco Tosete).

\section{Bibliografía}

Çelikbaş, Zeki. What is rss and how can it serve libraries?. Consultado en: 10-08-06. http://eprints.rclis.org/archive/00002531/01/ rss_and_libraries_EN3.pdf

Donoso, Rodrigo. "Blogs: herramientas de gestión del conocimiento". En: Biliotecnologías en acción. Bibliotecas digitales y blogs como herramientas de gestión del conocimiento, 2006. Consultado en: 16-09-06.

http://www.blogthink.cl/descargas/bcorp_rdonoso.pdf

Farkas, Meredith G. Wiki: the ultimate tool for online collaboration. Consultado en: 16-10-06. http://www.sirsidynixintitute.com/archive.php

Herrera Varela, Ricardo. "Bibliotecas públicas, incorporando nuevos servicios: el caso de los blogs institucionales". En: Pez de plata: bibliotecas públicas a la vanguardia, 2005, n. 3. Consultado en: 24-10-06.

http://eprints.rclis.org/archive/00005356/01/colaboracion_4.pdf

O'Reilly, Tim. Qué es web 2.0: patrones del diseño y modelos de negocio para la siguiente generación del software. Consultado en: 12-10-06. http://ociedaddelainformacion.telefonica.es/jsp/ articulos/detalle.jsp?elem $=2146$

Pérez Esteban, Mónica. Guía fácil del rss. Consultado en: 15-10-06.

http://es.geocities.com/rss_guia_facil/

Leticia Paula Dobrecky, Secretaría de Agricultura, Ganadería, Pesca y Alimentos de la Nación, Centro de Documentación e Información Agropecuaria, República Argentina, Ciudad Autónoma de Buenos Aires.

ldobre@mecon.gov.ar 